\title{
The heavy quark-antiquark potential from lattice and perturbative QCD
}

\author{
Alexander Laschka*, Norbert Kaiser and Wolfram Weise \\ Physik-Department, Technische Universität München, D-85747 Garching, Germany \\ E-mail: alaschka@ph.tum.de, nkaisereph.tum.de, weiseaph.tum.de
}

The heavy quark-antiquark potential in perturbative QCD is subject to ambiguities. We show how to derive a well-defined and stable short-distance potential that can be matched to results from lattice QCD simulations at intermediate distances. The static potential as well as the order $1 / \mathrm{m}$ potential are discussed.

8th Conference Quark Confinement and the Hadron Spectrum September 1-6, 2008

Mainz. Germany

\footnotetext{
* Speaker.
} 
The static quarkonium potential has been studied by lattice simulations as well as in perturbative QCD. It is an ideal object for exploring the interplay between perturbative and non-perturbative physics. However, the perturbative prediction tends to fail already at very small distances. It was found that this behaviour can be understood in the context of renormalons [1].

At two-loop order the static potential reads in momentum space [2]

$$
\tilde{V}^{(0)}(|\vec{q}|)=-\frac{4 \pi C_{F} \alpha_{s}(|\vec{q}|)}{\vec{q}^{2}}\left\{1+\frac{\alpha_{s}(|\vec{q}|)}{4 \pi} a_{1}+\left(\frac{\alpha_{s}(|\vec{q}|)}{4 \pi}\right)^{2} a_{2}+\ldots\right\},
$$

where $\vec{q}$ is the three-momentum transfer. Higher order terms involving IR divergences are not considered at this point. We define the static potential in coordinate space by a restricted Fourier transform with a low-momentum cutoff $q_{\text {min }}$ :

$$
V^{(0)}(|\vec{r}|)=\int_{|\vec{q}|>q_{\min }} \frac{d^{3} q}{(2 \pi)^{3}} e^{i \vec{q} \cdot \vec{r}} \tilde{V}^{(0)}(|\vec{q}|) .
$$
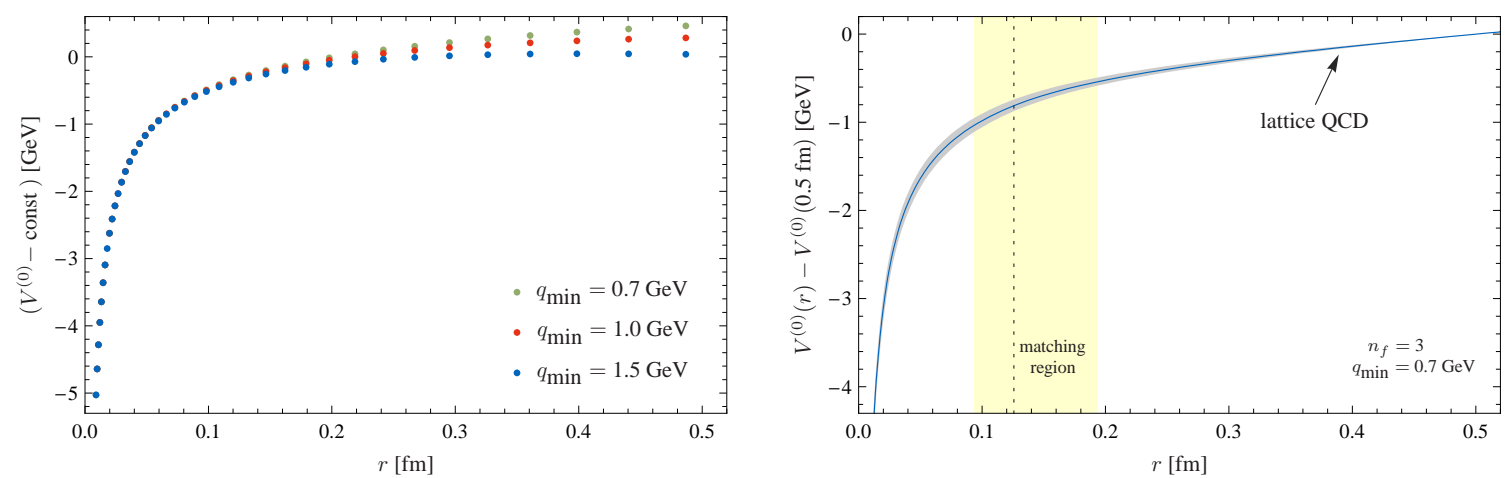

In contrast to the usual approach, the running coupling $\alpha_{s}$ is not expanded in a power series, but full four-loop RGE dependence in included in the transformation to coordinate space. The resulting potential depends only weakly on the cutoff (left figure) and can be matched at distances $r$ between 0.1 and $0.2 \mathrm{fm}$ to a potential obtained from lattice QCD [3]. The error band of the curve in the right figure reflects uncertainties in the Sommer scale $r_{0}=0.50 \pm 0.03 \mathrm{fm}$ (lattice part) and uncertainties in the scale dependence of $\alpha_{s}(|\vec{q}|)$ (perturbative part). The order $1 / \mathrm{m}$ potential can be defined analogously and can also be matched well to calculations from lattice QCD [4].

Work supported in part by BMBF, GSI and by the DFG Excellence Cluster "Origin and Structure of the Universe".

\section{References}

[1] M. Beneke, Phys. Lett. B434, 115 (1998) [hep-ph/9804241]; A. H. Hoang et al., Phys. Rev. D59, 114014 (1999) [hep-ph/9804227].

[2] M. Peter, Phys. Rev. Lett. 78, 602 (1997) [hep-ph/961020 9]; M. Peter, Nucl. Phys. B501, 471 (1997) [hep-ph / 9702245$]$; Y. Schröder, Phys. Lett. B447, 321 (1999) [hep-ph/98122 05].

[3] G. S. Bali et al., Phys. Rev. D62, 054503 (2000) [hep-lat/ 0003012 ].

[4] Y. Koma, M. Koma, and H. Wittig, PoS (LATTICE 2007) 111; M. Koma, PoS (Confinement 8) 105 (this conference). 